\title{
REVIEW
}

\section{From oxidative DNA damage to molecular epidemiology}

\author{
Katarína Volkovová ${ }^{1}$, Mária Dušinská ${ }^{1}$, Andrew R. Collins ${ }^{2}$ \\ ${ }^{1}$ Research Base of Slovak Medical University, Limbova 12, 85102 Bratislava, Slovakia \\ ${ }^{2}$ Institute for Nutrition Research, University of Oslo, Oslo, Norway
}

Received $20^{\text {th }}$ July 2005.

Published online $15^{\text {th }}$ November 2005.

\begin{abstract}
Summary
Reactive forms of oxygen are released during normal respiration, by the oxidative burst of the macrophages in response to infection, and by a variety of exogenous environmental agents. Oxidative stress, caused by imbalance between the production of reactive forms of oxygen and their elimination, leads to oxidative damage of biomolecules. It is generally accepted that oxidative stress is involved either as cause or effect in a variety of human degenerative diseases. The antioxidant hypothesis proposes that natural antioxidants in fruit and vegetables scavenge free radicals before they can cause damage. To test the hypothesis a reliable biomarker for in vivo assessment of oxidative damage is needed which can be correlated with antioxidant levels on one hand and with disease on the other. Oxidative damage to lymphocyte DNA can be measured by the comet assay or by HPLC. This paper gives a brief review of the methods and their application both in in vitro and in in vivo studies dealing with mutagenesis and carcinogenesis. Molecular epidemiological studies in Slovakia concerning different disease states, aging and the role of diet, and antioxidant supplementation in prevention are reviewed as well.
\end{abstract}

Keywords: oxidative damage - comet assay - DNA repair genes

\section{INTRODUCTION}

It seems to be generally accepted that oxidative stress is an inevitable feature of life, induced by reactive forms of oxygen released during normal respiration, by the oxidative burst of the macrophages in response to infection, and by a variety of exogenous agents including cigarette smoke and ionising radiation. It is also agreed that oxidative stress is something to be avoided, as it may be a contributing cause in a variety of human

Katarína Volkovová, Research Base of Slovak Medical University, Limbova 12, 85102 Bratislava, Slovakia

모․ katarina.volkovova@szu.sk degenerative diseases. The presence of powerful antioxidant defences, such as catalase, glutathione and associated enzymes within the cell points to the potential importance to the organism of protecting biomolecules against damage. Oxidation of DNA may lead to mutation (and hence to carcinogenesis); the most common altered base, 8oxo-guanine, can pair with $\mathrm{A}$ rather than $\mathrm{C}$, and so if it is present during replication, $\mathrm{C}>\mathrm{A}$ transversions may result. Oxidative stress is involved either as cause or effect in many human diseases. Peroxidation of lipids is thought to be a factor in the development of atherosclerotic plaques.

Not surprisingly, the 'antioxidant hypothesis' has arisen - and has been accepted virtually without question - as an explanation of the epidemiological link between consumption of fruit and vegetables and decreased risks of both 
cardiovascular disease and cancer. The hypothesis proposes that it is the natural antioxidants in fruit and vegetables - vitamins $\mathrm{C}$ and $\mathrm{E}$, carotenoids, flavonoids, etc. - that are protective, and that they act by scavenging reactive oxygen free radicals before they can cause damage. To test the hypothesis, we need a biomarker for in vivo oxidative stress which can be correlated with antioxidant levels on the one hand and with disease on the other.

In the ESCODD project we were comparing two methods for the detection of oxidative damage to lymphocyte DNA. We measured oxidative DNA damage by the comet assay or by HPLC. This biomarker has been used to investigate the protective effects of antioxidants in vitro, and to estimate levels of oxidative damage in various disease states and in the aging process.

\section{THE COMET ASSAY (SINGLE CELL GEL ELECTROPHORESIS)}

Cells are embedded in agarose on microscope slides and lysed with Triton X-100 and $2.5 \mathrm{M}$ $\mathrm{NaCl}$, which leaves the DNA, stripped of most protein, as nucleoids. The slides are placed in alkaline solution prior to electrophoresis. The DNA, being supercoiled, resists electrophoresis; however, breaks in loops of DNA relax supercoils in those loops, which are then free to migrate. They form a 'tail' to the nucleoid 'head' (hence the designation of 'comet'), and the percentage of DNA that moves into the tail reflects quantitatively the frequency of DNA breaks, over a range from a few hundred per cell up to several thousand (Ross et al. 1994). The assay for DNA breaks is of limited use in looking for endogenous oxidative damage to DNA, since breaks can arise in a variety of ways unrelated to oxidation, and furthermore breaks are quite rapidly repaired by cells. We have therefore modified the assay by incorporating a step, between lysis and electrophoresis, at which the DNA is digested with a bacterial repair endonuclease specific for certain kinds of DNA lesion (Collins et al. 1996). Endonuclease III nicks DNA at sites of oxidised pyrimidines; formamidopyrimidine glycosylase (fpg) recognises altered purines including 8-oxo-guanine and nicks the DNA alongside (Collins et al. 1997). By measuring the increase in tail DNA in the presence of one of these enzymes, we can estimate the amount of oxidative base damage in a sample of cells (8-oxo-guanine is probably the principal substrate for fpg in vivo). After staining with DAPI (a fluorescent dye which binds to DNA), relative tail intensity is assessed either visually, by sorting 100 comets into classes from 0 (no detectable tail) to 4 (large tail, minimal head), giving an overall damage score of $0-400$; or by computer image analysis, which gives the mean $\%$ of DNA in the tail. The scoring methods are equally reliable, and either kind of score can be expressed in terms of actual DNA break frequency by use of a calibration curve established with ionising radiation to introduce known numbers of DNA breaks. Thus we have been able to estimate the normal amount of oxidised pyrimidines and altered purines in a sample of 30 healthy male volunteers at around 1000 of each per cell. It should be appreciated that this measured damage is a steady state level, representing the balance between input and repair, both of which are presumably continuously occurring.

\section{8-OXO-DG MEASURED BY HPLC}

A more conventional method of measuring oxidative damage to DNA is to assay directly for 8oxo-dG in hydrolysed DNA, using HPLC with electrochemical detection, or to analyse for 8-oxoguanine with GC-MS. Reported values for endogenous damage in white blood cell DNA vary enormously - over at least two orders of magnitude (ESCODD 2002); oxidation during isolation and preparation of the DNA hydrolysate for analysis can account for some of the discrepancies, and recently developed methods attempt to eliminate this artefact, and produce lower values for endogenous damage (ESCODD 2003). On the same 30 samples that were analysed with the comet assay, we found a concentration of 8-oxo-dG equivalent to over 10,000 per cell, and so there is clearly an unresolved problem; either the enzymic methods (the comet assay, and other methods where damaged bases are converted to DNA breaks) underestimate damage, or the spurious oxidation of $\mathrm{dG}$ is still affecting analysis by HPLC. In spite of this puzzling state of affairs, both approaches - the comet assay with oxidationspecific endonucleases, and HPLC - are apparently valid indicators of oxidative stress.

\section{MEASUREMENT OF OXIDATIVE DNA DAMAGE AND THE PROTECTIVE EFFECT OF ANTIOXIDANTS IN VITRO}

Living organisms possess a variety of selfprotective mechanisms which decrease the free radical attack on DNA and so reduce the risk of cancer and other diseases. The protection of DNA by endogenous antioxidant systems may be significantly increased by numerous exogenously administered antioxidants, many of them representing important dietary factors. To this group belong lignins - polyphenols present in all 
plants. The comet assay method was used to show that pre-incubation of human VH10 cells and hamster V79 cells with lignin or vitamin E, reduced mainly the level of oxidised pyrimidines (Slamenova et al. 1999). In addition, lignin exhibited a protective effect against N-methyl-N'nitrosoguanidine (MNNG) - induced DNA damage, whereas vitamin $\mathrm{E}$ exhibited a protective effect only against $\mathrm{H}_{2} \mathrm{O}_{2}$-induced DNA damage. Moreover, it was shown, that the antimutagenic effect of lignin A against MNNG is associated with both adsorptive and antioxidative action. The presence of lignin A neither helped to renew DNA replication nor influenced the kinetics of DNA rejoining in MNNG-treated V79 cells (Labaj et al. 2003). In vivo experiments confirmed the antioxidative and antimutagenic properties of the biopolymer lignin: given to Sprague-Dawley rats in diet, lignin caused a significant decrease of H2O2induced, and visible light induced oxidative DNA lesions in freshly isolated rat lymphocytes, although, as in in vitro experiments, it did not influence the kinetics of rejoining of DNA strand breaks (Labaj et al. 2004).

Slamenova et al. (2002) studied the effect of dietary intake of vitamin A or $\mathrm{E}$ on genetic stability. Hepatocytes freshly isolated from male Wistar rats fed a common diet or a vitamin A- or Esupplemented diet were assayed for sensitivity to DNA breakage and cytogenetic changes induced by different indirectly acting carcinogens. In hepatocyte from rats fed vitamin A they observed a reduction in the severity of damage after $\mathrm{N}$ nitrosomorpholine treatment; after benzo(a)pyrene treatment there was a reduction in DNA breaks and chromosome aberrations; and after dimethyldibenzo[c,g]carbazole treatment they observed a reduction in DNA breaks. Treatment with vitamin $\mathrm{E}$ was less effective, although both vitamins reduced DNA strand breaks induced by the oxidative effect of a visible light-excited photosensitizer.

Gabelova and Pleskova (2000) tested the scavenging ability of carboxymethylglucan (CMG) on hydrogen peroxide or methylene blue plus visible light induced oxidative DNA damage in CaCo- 2 cells. While DNA lesions generated by hydrogen peroxide dominated by strand breakage, exposure of $\mathrm{CaCo}-2$ cells to visible light led mainly to base modifications sensitive to formamidopyrimidine DNA-glycosylase. Pretreatment of cells with ascorbic acid, a known anitoxidant, resulted in a statistically significant reduction of DNA lesions induced by $\mathrm{H}_{2} \mathrm{O}_{2}$ or visible light. However, pretreatment of $\mathrm{CaCo}-2$ cells with $\mathrm{CMG}$ in concentrations ranging from $0.01 \mu \mathrm{M}$ to $1 \mu \mathrm{M}$ did not give positive results.

The dietary effect of carboxymethyl chitinglucan (CM-CG) was examined in ex vivo experiments (Lazarova et al. 2004): Lymphocytes, testicular cells, alveolar macrophages and epithelial II cells were isolated from Sprague Dawley rats fed a common or CM-CG enriched diet, were freshly isolated and in in vitro conditions exposed to $\mathrm{H}_{2} \mathrm{O}_{2}$. A dose dependent increase of DNA breaks was observed after $\mathrm{H}_{2} \mathrm{O}_{2}$ treatment in all studied cell types. The number of DNA breaks in cells isolated from CM-CG supplemented animals was lower compared with the control animals fed a common diet.

\section{OXIDATIVE DNA DAMAGE IS LINKED TO DIETARY ANTIOXIDANT LEVELS}

We have recently used the comet assay to measure DNA damage (strand breaks, oxidised pyrimidines and altered purines) in lymphocytes from middle aged men taking part in a supplementation trial. They received a mixture of antioxidants: $100 \mathrm{mg}$ of vitamin $\mathrm{C}, 100 \mathrm{mg}$ of vitamin $\mathrm{E}, 6 \mathrm{mg}$ of $\beta$ carotene and $50 \mu \mathrm{g}$ of selenium or a placebo for 12 weeks. Plasma concentrations of these and other dietary antioxidants were measured by HPLC at week 0 (before supplementation) and week 12 (at the end of supplementation). We found that a combined antioxidant supplementation is effective even at these very moderate doses: it effectively decreases chromosomal instability in lymphocytes of middle-aged men (Dušinská et al. 2003, Volkovová et al. 2005).

\section{VEGETARIAN STUDY}

With the aim of studying the possible role of different diets in maintaining genomic stability we measured chromosome aberrations, and micronuclei and DNA damage in peripheral blood lymphocytes of vegetarians and non-vegetarians. The results of the study indicate that a vegetarian diet can lead to a slight decrease in oxidative DNA damage in lymphocytes, but other markers of genetic stability were not affected (Kažimírová et al. 2004).

\section{OXIDATIVE DAMAGE IS ELEVATED IN DISEASE}

Diabetes also belongs to diseases associated with oxidative stress, and oxidative DNA damage promises to be a useful biomarker of possible use in diagnosis, monitoring of treatment, and aetiological investigations. We measured DNA strand breaks, endonuclease III- and fpg-sensitive sites in lymphocytes from 10 patients with insulin- 
dependent diabetes mellitus; compared with an ageand sex-matched control group, the mean level of strand breaks, and of oxidised pyrimidines, were significantly elevated. A strong correlation was seen between fpg-sensitive sites and serum glucose concentration. DNA damage in lymphocytes is thus a useful marker of oxidative stress, and in particular fpg-sensitive sites seem to represent changes specifically related to hyperglycemia (Collins et al. 1998).

\section{ROLE OF OXIDATIVE DAMAGE IN AGING}

Oxidative damage to cellular structure and function is thought to be associated with the aging process and age-related diseases. Recently we have conducted a study to investigate aging using a molecular epidemiological and multidisciplinary approach. Two hundred and ninety one subjects were recruited to participate, 151 young people aged $20-25$ years and 140 elderly people aged $65-$ 70 years. The investigated subjects were relatively healthy volunteers without any acute disease or diabetes. We did not detect more DNA damage in isolated lymphocytes of old subjects than in young people. Chromosome aberrations, and, even more, micronuclei increase with age and can be regarded as a good biomarker of aging.

\section{OXIDATIVE DNA DAMAGE AS A BIOMARKER IN HUMAN BIOMONITORING STUDIES}

To compare the genotoxic effects of asbestos and rockwool fibre, a molecular epidemiological study was conducted in a former asbestos cement plant and rockwool plant in Slovakia. Biomarkers of exposure, effect and individual susceptibility were measured, including DNA damage (strand breaks (SBs), base oxidation and alkylation, using the comet assay), cytogenetic parameters and individual repair capacity (incision at 8-oxoguanine measured using a modified comet assay). We found significantly higher oxidised pyrimidines in subjects exposed to asbestos, and oxidised pyrimidines and alkylated bases strongly correlated with years of occupational exposure. Moreover, exposed asbestos workers had significantly higher numbers of chromosomal aberrations compared with the control group (Dušinská et al. 2004). There was no effect of rockwool exposure on specific damage to bases in DNA, nor on chromosome aberrations. DNA repair (8oxoguanine DNA glycosylase activity) was uneffected by exposure, but was negatively correlated with micronucleus frequency, implying that unrepaired 8-oxoguanine contributes to micronucleus formation (Dušinská et al. 2004).

\section{BIOMARKERS OF INDIVIDUAL SUSCEPTIBILITY TO CANCER AND OTHER DISEASES}

Sequence variations in a number of genes for DNA repair and phase I/phase II metabolism have recently been applied in human biomonitoring studies as putative biomarkers of individual susceptibility to cancer and possibly other diseases, measured alongside markers of exposure and effect. This molecular epidemiological approach will, we hope, allow us to assess the potential risk of environmental exposure and other factors at the level of individuals.

We recently conducted a biomonitoring study in 3 factories in Slovakia (asbestos, glass fibres and rockwool). Altogether 387 subjects (239 exposed, 148 controls) were investigated. Polymorphisms in five repair genes were determined by PCR-RFLP assays: XRCC1 (exon 10, G/A, Arg399Gln); XPD ( exon 10, G/A, Asp312Asn and exon 23, A/C, Lys751Gln); XPA (5' non-coding region, $23 \mathrm{~A} / \mathrm{G})$; and $\mathrm{O}^{6}$-methylguanine-DNA methyltransferase (MGMT, promotor-enhancer, $1099 \mathrm{C} / \mathrm{T})$. We also measured DNA damage (strand breaks [SBs], base oxidation and alkylation, using the comet assay); individual DNA repair capacity in lymphocyte extracts; micronuclei and chromosome aberrations; cellular defences (intrinsic antioxidants, antioxidant enzymes); humoral and cellular immune markers, growth factors and proinflammatory mediators. We found many interesting interactions between genetic variation in biotransfomation enzymes and parameters of oxidative stress and antioxidant defence especially between $G S T$ genotype and vitamin C levels. The association found in many subgroups suggests that the GST genotypes may affect vitamin C levels and thus may contribute to individual susceptibility and variation in response to endogenous and exogenous factors.

The XRCC1 protein is involved in base excision repair; it is therefore not surprising that we found a relationship with oxidative DNA damage. Subjects homozygous in the wild type allele $\mathrm{Arg} / \mathrm{Arg}$ for XRCC1 had significantly lower levels of oxidised purines compared with both GT heterozygotes and TT homozygotes. This association was seen in asbestos-exposed subjects as well as in the whole group $(\mathrm{p}=0.003, \mathrm{p}=0.036)$. An association of SBs with $X R C C 1$ variants was seen in the glass fibre factory.

Although the XPA protein is important in nucleotide excision repair, we found strong links between the variant XPA allele and elevated 
oxidative DNA damage, in all subjects $(\mathrm{p}=0.016$, $\mathrm{n}=354$ ), as well as in sub-groups - women $(\mathrm{p}=0.018, \mathrm{n}=150$, both oxidised purines and pyrimidines $)$, men $(\mathrm{p}=0.0041, \mathrm{n}=222)$ all controls $(\mathrm{p}=0.05, \mathrm{n}=131)$, exposed women $(\mathrm{p}=0.004, \mathrm{n}=75)$, exposed men $(\mathrm{p}=0.009, \mathrm{n}=157)$, and non-smokers $(\mathrm{p}=0.015, \mathrm{n}=238)$. The associations found in our study between repair gene polymorphisms and DNA instability imply a role for this group of genes in modulating the effects of exposure.

Measurement of oxidative damage (either in DNA or lipids ) seems to be an important biomarker. Its importance and implication in cancer and other diseases can be extensively studied in molecular epidemiological studies.

\section{REFERENCES}

Collins A.R., Dušinská M., Gedik C.M., Štetina R.: Oxidative damage to DNA: do we have a reliable biomarker? Environ. Health Perspect. 104:465-469, 1996.

Collins A.R., Dušinská M., Franklin M. et al.: Comet assay in human biomonitoring studies: reliability, validation and applications Environ. Mol. Mutagen. 30:139-146, 1997.

Collins A.R., Raslova K., Somorovska M. et al.: DNA damage in diabetes: correlation with a clinical marker. Free Radic. Biol. Med. 25:373-377, 1998.

Dušinská M., Kažimírová A., Barančoková M. et al.: Nutritional supplementation with antioxidants decreases chromosomal damage in humans. Mutagenesis 18:371-376, 2003.

Dušinská M., Collins A.R., Kažimírová A. et al.: Genotoxic effects of asbestos in humans. Mutat. Res. 553:91-102, 2004.

Dušinská M., Barančoková M., Kažimírová A. et al.: Does occupational exposure to mineral fibres cause DNA or chromosome damage? Mutat. Res. 553:103-110, 2004.

ESCODD (European Standards Committee on Oxidative DNA Damage): Comparative analysis of baseline 8-oxo-7,8dihydroguanine in mammalian cell DNA, by different methods in different laboratories: an approach to consensus. Carcinogenesis 23:2129-33, 2002.

European Standards Committee on Oxidative DNA Damage (ESCODD): Measurement of DNA oxidation in human cells by chromatographic and enzymic methods. Free. Radic. Biol. Med. 15: 1089-1099, 2003.

Gabelova A., Pleskova M.: Failure of carboxymethylglucan to inhibit oxidative DNA damage induced by hydroxyl radicals or singlet oxygen. Neoplasma 47:354-361, 2000.

Kažimírová A., Barančoková M., Volkovová K. et al.: Does a vegetarian diet influence genomic stability? Eur. J. Nutr. 43:32-38, 2004.

Labaj J., Slamenov D., Kosikova B.: Reduction of genotoxic effects of the carcinogen $\mathrm{N}$ methyl-N'-nitro-N-Nitrosoguanidine by dietary lignin in mammalian cells cultured in vitro. Nutr. Cancer. 47:95-103, 2003.

Labaj J., Wsolova L., Lazarova M. et al.: Repair of oxidative DNA lesions in blood lymphocytes from Sprague-Dawley rats; the influence of dietary intake of lignin. Neoplasma 51:450-455, 2004.

Lazarova M., Labaj J., Kovacikova Z., Slamenova D.: Diet containing fungal (1-->3)-beta-Dglucan derivative exhibits protective effects against DNA lesions induced in freshly isolated rat cells. Neoplasma 51:431-435, 2004.

Ross G.M., McMillan T.J., Wilcox P., Collins A.R.: The single cell microgel electrophoresis assay (comet assay): technical aspects and applications. Report on the 5th LH, Gray Trust Workshop, Institute of Cancer Research, 1994. Mutat. Res. 337:57-60, 1995.

Slamenova D., Chalupa I., Robichova S. et al.: Effect of dietary intake of vitamin A or $\mathrm{E}$ on the level of DNA damage, chromosomal aberrations, and micronuclei induced in freshly isolated rat hepatocytes by different carcinogens. Nutr. Cancer. 42:117-124, 2002.

Slamenova D., Horvathova E., Kosikova B. et al.: Detection of lignin biopolymer- and vitamin E-stimulated reduction od DNA strand breaks in H2O2- and MNNG-treated mammalian cells by the comet assay. Nutr. Cancer. 33:88-94, 1999.

Volkovová K., Barančoková M., Kažinírová A. et al.: Antioxidant supplementation reduces inter-individual variation in markers of oxidative damage. Free Radic. Res. 39:659666, 2005. 